\title{
Age and fecal microbial strain-specific differences in patients with spondyloarthritis
}

\author{
Matthew L. Stoll ${ }^{1 *}$, Pamela F. Weiss ${ }^{2}$, Jennifer E. Weiss ${ }^{3}$, Peter A. Nigrovic ${ }^{4}$, Barbara S. Edelheit ${ }^{5}$, S. Lou Bridges Jr ${ }^{1}$, \\ Maria I. Danila ${ }^{1}$, Charles H. Spencer ${ }^{6}$, Marilynn G. Punaro ${ }^{7}$, Kenneth Schikler ${ }^{8}$, Andreas Reiff', Ranjit Kumar ${ }^{1}$, \\ Randy Q. Cron', Casey D. Morrow ${ }^{1}$ and Elliot J. Lefkowitz ${ }^{1}$
}

\begin{abstract}
Background: Prior studies have demonstrated abnormalities in the composition of the gastrointestinal microbiota in pediatric and adult patients with spondyloarthritis (SpA). In particular, diminished fecal abundance of Faecalibacterium prausnitzii and abnormalities in both directions in the abundance of the Bacteroides genus have been identified.

Methods: We obtained fecal specimens from 30 children with treatment-naïve enthesitis-related arthritis (ERA) and 19 healthy controls, as well as specimens from 11 adult patients with longstanding SpA and 10 adult healthy controls. All of the samples underwent sequencing of the $16 \mathrm{~S}$ ribosomal DNA. A subset of the pediatric fecal samples was subjected to shotgun metagenomics sequencing.

Results: ERA patients had decreased abundance of the anti-inflammatory F. prausnitzii A2-165 strain $(41 \pm 28 \%$ versus 54 $\pm 20 \%$ of all sequences matching F. prausnitzii, $p=0.084$ ) and an increased abundance of the control F. prausnitzii L2/6 strain $(28 \pm 28 \%$ versus $15 \pm 15 \%, p=0.038)$. Similar trends were observed in adults with longstanding SpA $(n=11)$ and controls $(n=10)$. In contrast, the fecal abundance of Bacteroides fragilis was increased in ERA subjects $(2.0 \pm 4.0 \%$ versus 0 . $45 \pm 0.7 \%$ of all sequences, $p=0.045)$, yet was diminished in adult subjects $(0.2 \pm \%$ versus $1.0 \pm \%$ of all sequences, $p=0$. 106). Shotgun metagenomics sequencing of the fecal DNA in the pediatric subjects revealed diminished coverage of the butanoate pathway (abundance normalized to controls of $1 \pm 0.48$ versus $0.72 \pm 0.33$ in ERA, $p=0.037$ ).
\end{abstract}

Conclusions: The anti-inflammatory F. prausnitzii A2-165 strain appears to be depleted in both pediatric and adult SpA. In contrast, B. fragilis may be depleted in adult disease yet abundant in pediatric SpA, suggesting developmental effects on the immune system.

Keywords: Microbiota, Spondyloarthritis, Sequencing, Bacteroides, Faecalibacterium

\section{Background}

The role of the intestinal microbiota in the pathogenesis of spondyloarthritis $(\mathrm{SpA})$ is gaining widespread interest [1]. Much of this interest was spurred by a large body of literature indicating abnormalities of the microbiota in patients with inflammatory bowel disease (IBD) [2], a condition in which therapeutic alteration of the microbiota in the form of antibiotics, probiotics, and even fecal transplant may be effective [3], along with the clinical and genetic associations between IBD and SpA [4]. Prior studies evaluating fecal or

\footnotetext{
* Correspondence: mstoll@peds.uab.edu

${ }^{1}$ University of Alabama at Birmingham, Birmingham, AL, USA

Full list of author information is available at the end of the article
}

mucosal microbiota of adult patients with SpA demonstrated diminished fecal abundance of Bacteroides [5, 6], with one study showing a potential association between the Dialister genus and disease severity [6]. There are comparatively few data reported in pediatric SpA. One study showed decreased abundance of Prevotella in pediatric SpA [7], and our prior work showed decreased abundance of Faecalibacterium prausnitzii [8]. The latter finding is consistent with multiple similar reports in subjects with IBD [9]. Depletion of F. prausnitzii may adversely impact intestinal health through diminished production of butyrate and other short chain fatty acids [10], and our previous work has shown diminished abundance of butyrate in the fecal 
water of children with ERA [11]. Both pediatric SpA studies also showed increased abundance of unspecified species within the Bacteroides genus, consistent with studies in children with other categories of juvenile idiopathic arthritis (JIA) [12, 13], yet the opposite of findings in adult subjects with rheumatoid arthritis (RA) $[14,15]$ or SpA [5, 6].

Herein, we studied the intestinal microbiota in children with treatment-naïve $\mathrm{SpA}$ in comparison to pediatric healthy controls; to do this, we used a novel bioinformatics tool in combination with the Basic Local Alignment Search Tool (BLAST) that permitted assessment of species and even strain-level identification of certain organisms, as different strains within a species can have dramatically different effects [16, 17]. In order to validate our prior results and to further increase the sample size, we recruited subjects from geographic locations around the country. Prior studies have shown that geography can impact the microbiota in situations in which subjects with highly different environments (e.g., urban versus rural) and ethnicities are compared $[18,19]$, but there are no data evaluating subjects from different urban areas specifically within the United States. The findings in these subjects were compared with those in adults with longstanding SpA and adult controls, to identify which changes might be common to SpA and which might be unique to pediatric populations. We also performed shotgun metagenomics sequencing on a subset of children with juvenile $\mathrm{SpA}$ in order to obtain additional information regarding potential mechanisms whereby the microbiota might predispose to SpA. Herein, we demonstrate that the anti-inflammatory A2-165 strain of F. prausnitzii is depleted in both pediatric and adult SpA, and that the microbiota of children with SpA has decreased genetic capacity to synthesize butyrate. We additionally demonstrate that B. fragilis is depleted in adult SpA yet abundant in pediatric disease.

\section{Methods}

\section{Subjects}

Pediatric SpA subjects were mostly children with enthesitisrelated arthritis (ERA; juvenile SpA) as per the International League of Association for Rheumatology criteria [20]; three with sacroiliitis in the absence of peripheral arthritis met the Assessment of Spondyloarthritis International Society (ASAS) criteria for axial SpA [21]. Pediatric controls were either healthy children recruited through advertisements or children referred to rheumatology for evaluation of arthritis but found to have noninflammatory causes of joint pain or irrelevant laboratory markers, such as a positive antinuclear antibody. None of the controls had any findings suggestive of infectious arthropathies, including Lyme synovitis. Pediatric subjects were recruited from eight sites around the country (Table 1). Exclusion criteria were receipt of antibiotics within 3 months prior to study enrollment and prior treatment with any immunosuppressive agent excluding less
Table 1 Demographic and clinical characteristics of pediatric study participants

\begin{tabular}{|c|c|c|}
\hline Characteristic & ERA & Controls \\
\hline$n$ & 30 & 19 \\
\hline Age (years), mean $\pm \mathrm{SD}$ & $13.5 \pm 3.0$ & $13.6 \pm 2.7$ \\
\hline $\mathrm{BMI}\left(\mathrm{kg} / \mathrm{m}^{2}\right)$, mean $\pm \mathrm{SD}$ & $20.7 \pm 4.1$ & $21.5 \pm 6.1$ \\
\hline Male & 19 (63\%) & $13(68 \%)$ \\
\hline \multicolumn{3}{|l|}{ Race } \\
\hline Caucasian & $23(77 \%)$ & $17(89 \%)$ \\
\hline African-American & $3(10 \%)$ & $2(10 \%)$ \\
\hline Asian & $2(6.7 \%)$ & 0 \\
\hline Other/unknown & $2(6.7 \%)$ & 0 \\
\hline \multicolumn{3}{|l|}{ Site } \\
\hline UAB (Birmingham, AL, USA) & $8(27 \%)$ & $9(47 \%)$ \\
\hline CHOP (Philadelphia, PA, USA) & $10(33 \%)$ & $4(21 \%)$ \\
\hline HUMC (Hackensack, NJ, USA) & $1(3.3 \%)$ & $4(21 \%)$ \\
\hline CCMC (Hartford, CT, USA) & $1(3.3 \%)$ & $2(10 \%)$ \\
\hline BCH (Boston, MA, USA) & $4(13 \%)$ & 0 \\
\hline $\mathrm{NCH}$ (Columbus, OH, USA) & $2(6.7 \%)$ & 0 \\
\hline TSRH (Dallas, TX, USA) & $2(6.7 \%)$ & 0 \\
\hline CHLA (Los Angeles, CA, USA) & $1(3.3 \%)$ & 0 \\
\hline UL (Louisville, KY, USA) & $1(3.3 \%)$ & 0 \\
\hline $\mathrm{HLA}-\mathrm{B} 27^{+}$ & $15 / 29(52 \%)$ & Not measured \\
\hline Sacroiliitis & $18(60 \%)$ & None \\
\hline Clinical only & $8 / 18(44 \%)$ & \\
\hline Imaging & $10 / 18(56 \%)$ & \\
\hline
\end{tabular}

Data presented as $n(\%)$ unless stated otherwise

$B C H$ Boston Children's Hospital, BMI body mass index, CHLA Children's Hospital of Los Angeles, CCMC Connecticut Children's Medical Center, CHOP Children's Hospital of Philadelphia, ERA enthesitis-related arthritis, HUMC Hackensack University Medical Center, NCH Nationwide Children's Hospital, TSRH Texas Scottish Rite Hospital, UAB University of Alabama at Birmingham, UL University of Louisville

than 2 weeks of corticosteroids. None of the pediatric subjects or controls had been included in our prior work [8].

Adult SpA subjects were all recruited at the University of Alabama at Birmingham (UAB). Patients met either the ASAS criteria for peripheral [22] or axial [21] SpA or the Classification Criteria for Psoriatic Arthritis (CASPAR) criteria [23] for psoriatic arthritis (PsA). Sacroiliitis in both children and adults was defined based upon either clinical findings per the judgment of the treating rheumatologist or imaging findings on MRI or radiography; only a single observer was required. Adult controls were healthy volunteers recruited through advertisements or patients with noninflammatory causes of joint pain (Table 2). For the adult subjects, as with the pediatric counterparts, recent antibiotic use was an exclusion criterion; however, for the adult subjects, immunosuppressive therapy was not. 
Table 2 Demographic and clinical characteristic of adult study participants

\begin{tabular}{|c|c|c|}
\hline Characteristic & SpA & Controls \\
\hline$n$ & 11 & 10 \\
\hline Age (years), mean $\pm S D$ & $52 \pm 7.4$ & $47 \pm 8.1$ \\
\hline BMI $\left(\mathrm{kg} / \mathrm{m}^{2}\right)$, mean $\pm \mathrm{SD}$ & $32 \pm 7.8$ & $28 \pm 4.8$ \\
\hline Disease duration (years), mean \pm SD & $11 \pm 9.5$ & Not applicable \\
\hline Male & $4(36 \%)$ & $3(30 \%)$ \\
\hline \multicolumn{3}{|l|}{ Race } \\
\hline Caucasian & $9(82 \%)$ & $6(60 \%)$ \\
\hline African-American & $2(18 \%)$ & $4(40 \%)$ \\
\hline Diagnosis & & Not applicable \\
\hline Undifferentiated SpA & $1(9.1 \%)$ & \\
\hline Ankylosing spondylitis & $6(54 \%)$ & \\
\hline IBD-associated arthritis & $1(9.1 \%)$ & \\
\hline Psoriatic arthritis & $2(18 \%)$ & \\
\hline Reactive arthritis & $1(9.1 \%)$ & \\
\hline \multicolumn{3}{|l|}{ Current medications } \\
\hline None & $2(18 \%)$ & $10(100 \%)$ \\
\hline Traditional DMARDs alone & $5(45 \%)$ & \\
\hline TNFi alone & $3(27 \%)$ & \\
\hline DMARDs plus TNFi & $1(9.1 \%)$ & \\
\hline HLA-B27 $7^{+}$ & $3 / 3(100 \%)$ & Not measured \\
\hline Sacroiliitis & $8(73 \%)$ & None \\
\hline Clinical & $1(12 \%)$ & \\
\hline Imaging & $7(88 \%)$ & \\
\hline
\end{tabular}

Data presented as $n(\%)$ unless stated otherwise

$B M I$ body mass index, DMARD disease-modifying anti-rheumatic drug (leflunomide, $n=2$; methotrexate, $n=2$; sulfasalazine, $n=3$ ), IBD inflammatory bowel disease, SpA spondyloarthritis, TNFi tumor necrosis factor inhibitor

\section{Processing of fecal samples}

Subjects collected the samples at home and immediately placed the samples in a 50-ml container filled with CaryBlair media. Samples were shipped overnight via commercial carrier to the microbiota core at UAB. Microbial genomic DNA was isolated by standard methods using kits from Zymo Research (Irvine, CA, USA) as per the manufacturer's instructions. For 16S sequencing, the Zymo ZR Fecal DNA MiniPrep kit (catalog \# D6010) was used; for shotgun metagenomics, the whole genome DNA kit (catalog \# D6110) was used.

\section{Sequencing and analysis of $16 \mathrm{~S}$ ribosomal DNA from the fecal specimens}

The purified DNA ( 100 ng) underwent PCR amplification using primers designed to the conserved region flanking the V4 region from the $16 \mathrm{~S}$ ribosomal DNA (rDNA) gene, as described previously [8]. Resulting PCR fragments were run on the Illumina MiSeq (San
Diego, CA, USA) at a concentration of $12 \mathrm{pM}$; read lengths were approximately 250-bp paired-end reads. Initial steps of the analysis were performed with the Divisive Amplicon Denoising Algorithm (DADA2) [24, 25]. Unlike some of the widely used algorithms [26, 27], DADA2 does not cluster similar sequences. Instead, it uses error modeling to distinguish amplification or sequencing errors from true sequences, which are typically referred to as sequence variants (SVs). This assessment is based upon features of the sequence, specifically the distance from its nearest neighbor as measured by the number of nucleotide differences and as influenced by quality scores and specific nucleotide transitions; and its abundance. Thus, a highly abundant sequence may be assessed as a true SV rather than a sequence error, even if it is highly similar to another abundant SV. Consequently, DADA2 can identify unique strains within a species. The output of DADA2 is an abundance table, in which each unique sequence is characterized by its abundance in each sample. Taxonomic information was incorporated into this abundance table with the ribosomal database project naive Bayesian classifier [28] using the May 2013 version of Greengenes [29]. This abundance table has the same structure as a traditional operational taxonomic unit table that is the analytic unit in the Quantitative Insight Into Microbial Ecology (QIIME) tool suite [26]. Thus, it was incorporated into QIIME with the biom convert script, and assessment of alpha and beta diversity was performed within QIIME. Alpha diversity indexes of richness and evenness were assessed with the Chao1 and Shannon measures respectively; beta diversity was assessed with the Bray Curtis measure, visualized using principal coordinates analysis (PCoA). Variables assessed were presence versus absence of arthritis, and among the ERA patients were geographic location, presence versus absence of sacroiliitis, and HLA-B27 status. Geographic location was assessed both by looking at each site as a separate unit and by pooling into one region the four sites located in the northeast (Boston Children's Hospital, Connecticut Children's Medical Center, Hackensack University Medical Center, and Children's Hospital of Philadelphia), a geographic area that is similar in size to the catchment area of UAB. To identify specific strains within $F$. prausnitzii, the $16 \mathrm{~S}$ sequences were downloaded from the National Library of Medicine, and the SVs matching F. prausnitzii were aligned against the known sequences using BLAST. The code used for the $16 \mathrm{~S}$ sequence analysis is available in Additional file 1.

\section{Sequencing and analysis of shotgun sequencing from the fecal specimens}

The purified DNA was sheared and 500-600-bp fragments were purified by agarose gel electrophoresis. Illumina kits 
were used for adapter ligation followed by Illumina HiSeq, 125-bp paired-end sequencing. Approximately 25-30 million reads per sample were obtained from each sample. A second set was run on the Illumina MiSeq, yielding 3-7 million reads per sample, approximately 250-bp paired-end sequencing. Quality control of both sets was performed using PRINSEQ [30]. Alignment with the human genome to remove human reads was performed with Bowtie2 [31] using the very-sensitive mode. Conversion of fastq to fasta files was performed with the FASTX tool kit (http://hannonlab.cshl.edu/fastx_toolkit/commandline.html). The remainder of the analysis was performed with the HMP Unified Metabolic Analysis Network (HUMAnN V2) program [32]. A detailed description of the program is available online (http://huttenhower.sph.harvard.edu/humann2). Briefly, the input fasta files were aligned against a functionally annotated pan-genome database of over 4000 species, named ChocoPhlan by the HUMAnN2 investigators, using Bowtie2 [31]. Reads not mapped to ChocoPhlan were subsequently aligned against the UniProt universal proteins database [33] using double index alignment of nextgeneration sequencing data (DIAMOND) [34], a more efficient alternative to BLASTX. Pathway assignments were performed with MetaCyc [35]; all genes associated with a pathway must be present for the pathway to be considered a match. Gene and pathway abundances were normalized to the sequencing depth prior to any further analyses. Translation from UniProt to Kyoto Encyclopedia of Genes and Genomes (KEGG) [36] was performed with the map_ko_uniref50.txt.gz mapping file made available by the HUMAnN investigators. The output of HUMAnN2 is files containing the abundance of each gene as well as of each pathway, optionally normalized to the sequencing depth of each subject. The code used for the HUMAnN2 analysis is included in Additional file 1.

\section{Statistical analysis}

To evaluate whether the samples as a whole clustered according to nominal variables, the permutation multivariate analysis of variance (PERMANOVA) test was run against the distance matrix generated from the Bray Curtis test of beta diversity. The PERMANOVA test partitions a distance matrix among sources of variation (e.g., presence versus absence of arthritis) in order to describe the strength and significance that a categorical variable has in determining the variation of distances [37]. Comparisons in the abundance of specific taxa, as well as assessments of differences in alpha diversity, were performed with Student's $t$ test. Pairwise comparisons performed at each of the phylogenetic levels (e.g., phylum) were corrected for multiple comparisons with the Benjamini-Hochberg false discovery rate (FDR) test [38] with a significance threshold of 0.05 .

\section{Results}

Subjects

The demographic and clinical features of the pediatric subjects are summarized in Table 1 . All were naïve to immunosuppressive therapy, and none had been exposed to systemic antibiotics within 3 months prior to enrollment in the study. The patients and controls were well matched for age and BMI.

\section{$16 \mathrm{~S}$ sequencing}

A mean of 129,226 (range 39,741-249,043) paired-end reads were obtained from the 49 subjects. Following quality filtering, merging, and chimera removal, a total of 64,016 (range 23,942-105,497) reads were used. Among the 49 subjects, a total of 1402 unique sequences (sequence variants (SVs)) were obtained; as most of these were rare and unique to specific subjects, the range was 46-236 SVs/subject.

PCoA on the 49 subjects demonstrated clustering by diagnosis (ERA versus control; Fig. 1). This visually evident clustering was confirmed by the PERMANOVA test $(F=1.45, p=0.046)$. Among the ERA patients, no sources of variation were observed on the basis of any single variable, including geographic location (Bray Curtis $F=1.09, p=0.277$ ), HLA-B27 status (Bray Curtis $F=$ $0.902, p=0.652$ ), or the presence of sacroiliitis (Bray Curtis $F=0.471, p=1)$. Geographic location was assessed both by looking at each site as a separate unit and by pooling into one region the four sites located in the northeast

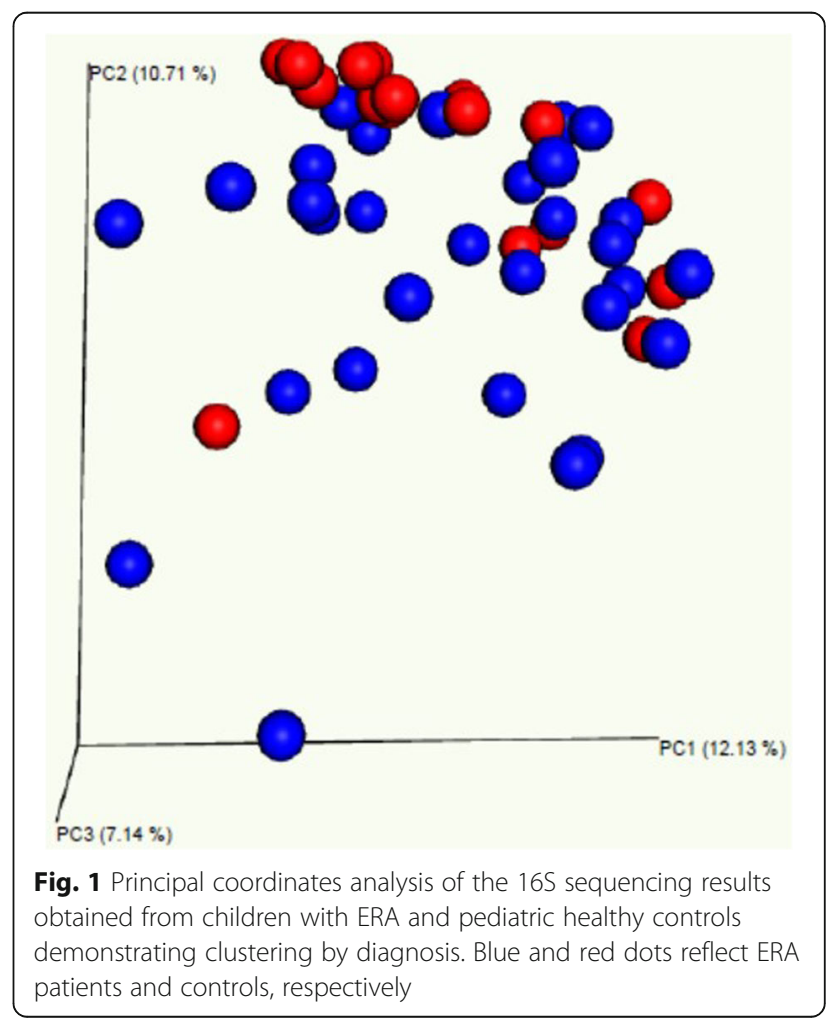


(Boston Children's Hospital, Connecticut Children's Medical Center, Hackensack University Medical Center, and Children's Hospital of Philadelphia), a geographic area that is similar in size to the catchment area of UAB.

Due to the large number of SVs as already indicated, pairwise comparisons at most of the phylogenetic levels failed to identify any groupwise differences following adjustment for multiple comparisons. The only exception was that ERA patients demonstrated decreased abundance of the Actinobacteria phylum as compared to controls (3.4\% versus $9.3 \%, p=0.002$ uncorrected, $p=0.05$ FDRcorrected). This lack of any significant pairwise differences held even after SVs within a species were collapsed into the species, thus reducing the number of unique items to 366. Consequently, based upon previous work in children with ERA [7, 8], the analysis was focused on two organisms: F. prausnitzii and the Bacteroides genus, particularly B. fragilis.

The abundance of $F$. prausnitzii as a whole was nominally higher in the patients (10 versus $7.8 \%, p=0.192$; Fig. 2a), in contrast to our previous results [8]. Twentythree unique SVs were identified within $F$. prausnitzii. As discussed in Methods, BLAST was used to identify strains within the $F$. prausnitzii SVs identified by DADA2. Of those $23 \mathrm{SVs}$, one of them was a $100 \%$ best match (253/253 bp) with strain A2-165, which has been shown previously to have regulatory effects [39]. Recent studies have demonstrated that even compared to other strains of $F$. prausnitzii, such as L2/6, the A2-165 strain induces increased interleukin-10 production from peripheral blood mononuclear cells $[16,40]$ and has increased butyrate promoter activity [41]. A separate SV was a $99 \%$ best match (252/253 bp) with L2/6, the comparator strain in some of the aforementioned studies. These two strains were the two most abundant SVs matching F. prausnitzii.
As a proportion of the sequences that matched $F$. prausnit$z i i$, ERA patients had relatively decreased abundance of the regulatory A2-165 strain $(41 \pm 28 \%$ versus $54 \pm 20 \%, p=$ 0.084; Fig. 2b) and a relatively increased abundance of the SV that most closely matched L2/6 (28 $\pm 28 \%$ versus $15 \pm$ $15 \%, p=0.038$; Fic 2c). Thus, the stool samples from pediatric ERA patients contained a decreased ratio of the antiinflammatory, relative to the neutral, strain of $F$. prausnitzii.

ERA patients and controls had similar levels of the Bacteroides genus (20 versus 19\%). However, among the Bacteroides genus, $B$. fragilis is considered to have regulatory properties through its polysaccharide tail [42] and thus was investigated further. Here, consistent with our previous findings [8], ERA patients had a four-fold increased abundance ( $2.0 \pm 4.0 \%$ versus $0.450 .7 \%, p=0.045$; Fig. $3 a)$. There were no significant differences with any of the other species of Bacteroides. In summary, children with ERA had diminished abundance of the regulatory A2-165 strain of $F$. prausnitzii as well as increased abundance of $B$. fragilis, compared to healthy control subjects.

To evaluate whether these strain-level and species-level differences between arthritis subjects and controls are unique to pediatric subjects or are potentially a marker of $\mathrm{SpA}$ as a whole, $16 \mathrm{~S}$ sequencing was run on a cohort of 11 adults with longstanding SpA and 10 healthy adult controls. Characteristics of the adult subjects are presented in Table 2; most had longstanding disease, with a mean disease duration of 11 years, and most were on immunosuppressive therapy. As with the pediatric subjects, none of the adult SpA patient or controls had been exposed to systemic antibiotics within 3 months of enrollment.

Among the adults, the opposite trend with respect to Bacteroides abundance was seen. Specifically, Bacteroides abundance as a proportion of total sequencing depth was
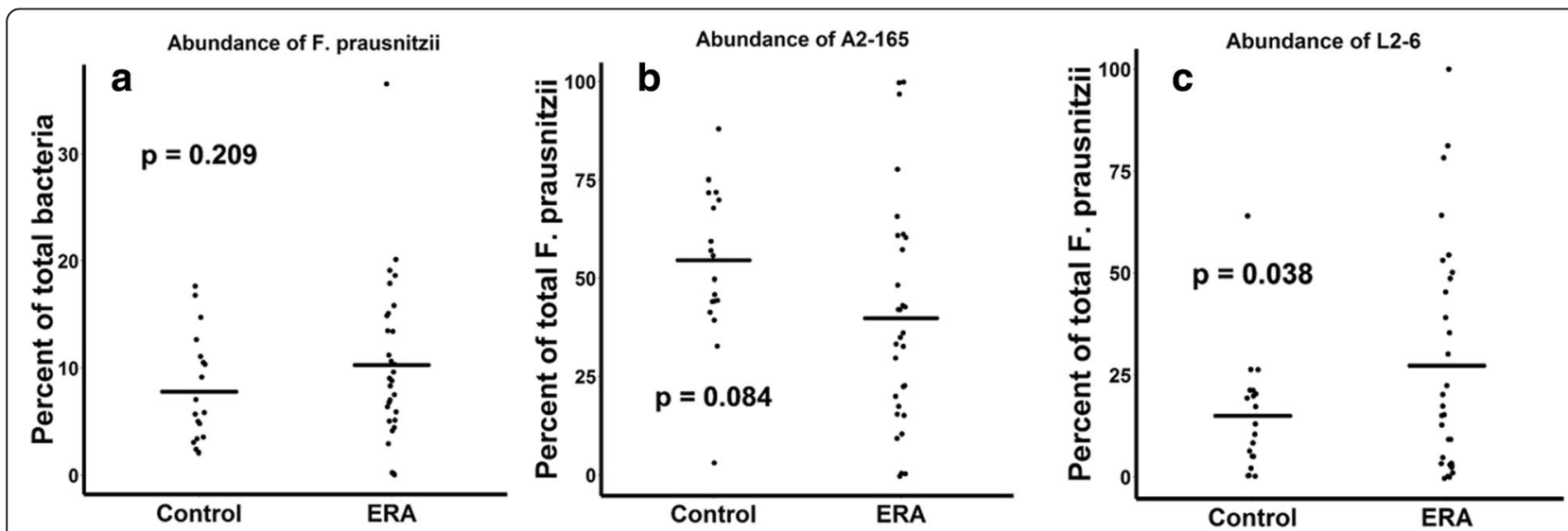

Fig. 2 Strain-level variation in the fecal abundance of Faecalibacterium prausnitzii in children with ERA and pediatric controls. Abundance of $F$. prausnitzii as a percentage of total fecal content is similar in patients and controls (a). As a percentage of total F. prausnitzii, children with ERA have a trend toward decreased fecal abundance of the anti-inflammatory A2-165 strain (b) as well as increased abundance of the L2/6 strain (c). ERA enthesitis-related arthritis 

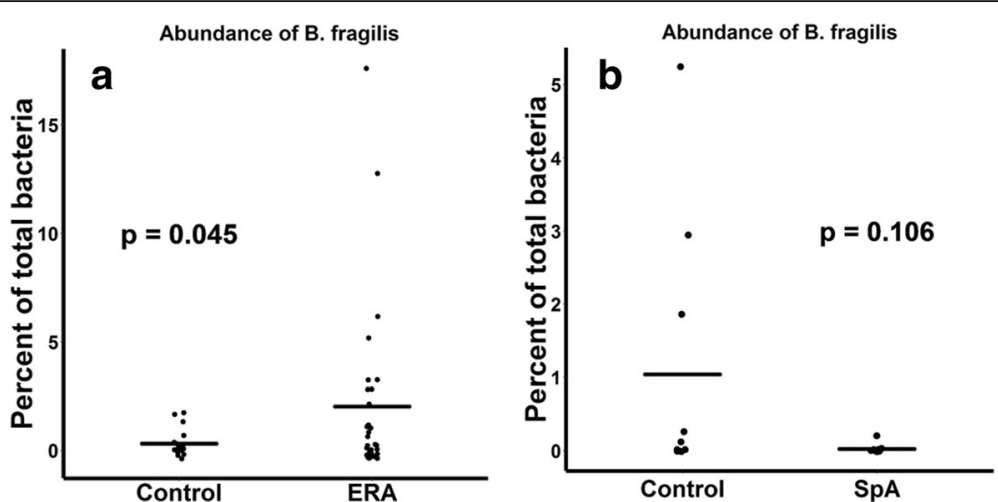

Fig. 3 Fecal abundance of Bacteroides fragilis in pediatric and adult subjects with SpA. Increased fecal abundance is observed in ERA patients compared to pediatric controls (a), while a trend toward decreased fecal abundance was observed in adult SpA patients compared to adult controls (b). ERA enthesitis-related arthritis, SpA spondyloarthritis

$11 \pm 9 \%$ among the patients, compared to $26 \pm 18 \%$ in the controls $(p=0.036)$, although there were no significant differences in the abundance of $B$. fragilis $(0.2 \pm 0.6 \%$ in patients versus $1 \pm 1.8 \%$ in controls, $p=0.106$ ) (Fig. $3 \mathrm{~b}$ ). In contrast, the trends involving $F$. prausnitzii strains were similar in adults as compared to children (total $F$. prausnitzii $6.9 \pm 6.9 \%$ in controls versus $10 \pm 10 \%$ in patients $(p$ $=0.427$ ); A2-165 as percentage of $F$. prausnitzii $41 \pm 30 \%$ in controls versus $25 \pm 13 \%$ in patients $(p=0.175)$ ), although the differences were not statistically significant, possibly due to a smaller sample size. Similar trends for both organisms were observed if the three subjects taking sulfasalazine were excluded (data not shown.)

Finally, the abundance of additional organisms that have emerged as being of interest in SpA was evaluated. Specifically, the Lachnospiraceae family was reduced in children with ERA in our previous work [8], yet was elevated in both biopsy [43] and fecal [44] specimens in prior studies of adults with SpA. There were, however, no differences in the abundance of this family in either the pediatric or the adult subjects (data not shown). A study of ileal biopsies demonstrated that the abundance of the Dialister genus correlated with disease activity in treatment-naïve SpA patients [6]; this organism was virtually undetectable $(<1 \%)$ in the fecal specimens of all subjects in both age groups, likely indicating specificity to mucosal specimens. Ruminococcus gnavus was elevated in the feces of adult subjects with SpA in a prior report [44], but the differences of this organism herein were not statistically significant in either age group. Finally, both Stebbings et al. [5] and our group [8] have shown increased abundance of Bifidobacterium in the feces of SpA patients; surprisingly, the same genus was depleted among pediatric ERA subjects compared to healthy controls $(2.6 \%$ versus $7.2 \%, p=0.008)$, with no differences among the adult subjects.

\section{Whole genome sequencing}

To assess the potential functional consequences of the presented findings, whole genome sequencing of fecal DNA with the HiSeq device was performed on a subset of the aforementioned pediatric patients, consisting of 14 patients each (ERA and healthy controls), supplemented by 12 additional subjects (five controls and seven ERA patients) who were sequenced with the MiSeq device. These subjects were similar with respect to demographic characteristics and BMI (Table 3). A mean of 23 million (range 14.7-38.7 million) high-quality sequences from the Illumina HiSeq sequencer was analyzed, as was a mean of 5.3 million (range 3.5-7.8 million) high-quality sequences from the MiSeq device. There was no obvious clustering based upon diagnosis at either the level of individual genes or whole pathways (data not shown), consistent with a core microbiome not affected by the disease state [45]. In light of our findings in the present study and our previous report [8] showing alterations in the fecal abundance of $F$. prausnitzii, we specifically examined the butanoate pathway (MetaCyc title CENTFERM-PWY pyruvate fermentation to butanoate), which results in synthesis of butyrate from precursors such as pyruvate and acetyl-CoA. With both devices, there was a modest difference in the abundance of this pathway. For both the MiSeq and HiSeq devices, each value was normalized to the mean value for the controls for the respective run, and the two sets were pooled, revealing a statistically significant decreased representation of the butanoate pathway in ERA patients compared to controls $(1 \pm 0.48$ versus $0.72 \pm 0.33, p=0.037$; Fig. 4.) Thus, our data indicate that the fecal microbiota of ERA patients compared to controls had decreased capacity to synthesize a compound that is generally considered to have antiinflammatory properties within the intestinal environment [46]. 
Table 3 Demographic and clinical characteristics of participants undergoing whole genome shotgun sequencing

\begin{tabular}{lll}
\hline Characteristic & ERA & Controls \\
\hline$n$ & 21 & 19 \\
Age (years), mean \pm SD & $13.6 \pm 3.0$ & $12.9 \pm 3.0$ \\
BMI (kg/m²), mean \pm SD & $21.0 \pm 5.1$ & $20.4 \pm 6.2$ \\
Male & $12(57 \%)$ & $12(63 \%)$ \\
Race & & \\
Caucasian & $14(67 \%)$ & $14(74 \%)$ \\
African-American & $5(24 \%)$ & $5(26 \%)$ \\
Asian & $2(9.5 \%)$ & 0 \\
Site & & \\
UAB (Birmingham, AL, USA) & $8(38 \%)$ & $11(58 \%)$ \\
CHOP (Philadelphia, PA, USA) & $7(33 \%)$ & $2(10 \%)$ \\
HUMC (Hackensack, NJ, USA) & $1(4.8 \%)$ & $2(10 \%)$ \\
CCMC (Hartford, CT, USA) & $1(4.8 \%)$ & $4(21 \%)$ \\
BCH (Boston, MA, USA) & $4(19 \%)$ & 0 \\
HLA-B27 ${ }^{+}$ & $10 / 20(50 \%)$ & Not measured \\
Sacroiliitis & $10(48 \%)$ & None \\
Clinical only & $4(40 \%)$ & \\
Imaging & $6(60 \%)$ & \\
\hline
\end{tabular}

Data presented as $n$ (\%) unless stated otherwise

$B C H$ Boston Children's Hospital, BMI body mass index, CCMC Connecticut Children's Medical Center, CHOP Children's Hospital of Philadelphia, ERA enthesitis-related arthritis, HUMC Hackensack University Medical Center, UAB University of Alabama at Birmingham

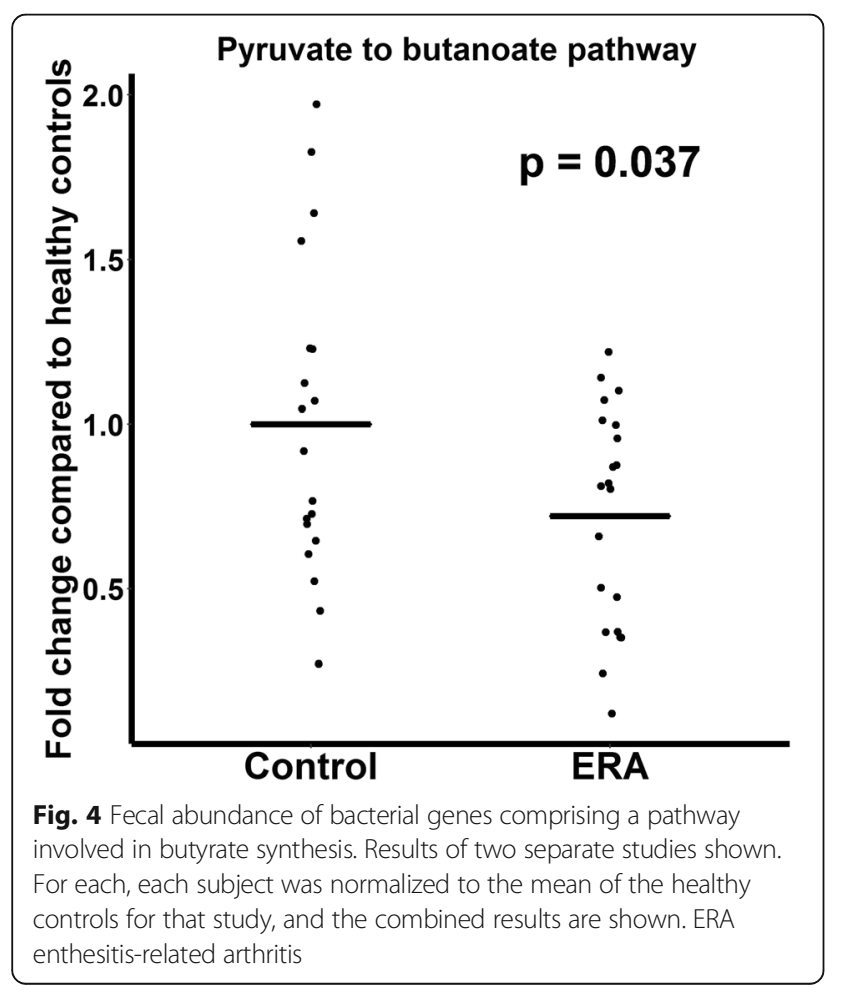

\section{Discussion}

Mechanisms by which an altered microbiota might be associated with inflammatory arthritis are as yet unknown. Animal studies clearly demonstrate a causal relationship, as an animal model of SpA is abrogated in the germ-free environment [47]. Herein, we report strain-level differences in fecal abundance of $F$. prausnitzii between children with ERA and healthy controls. Additionally, at the functional level, decreased genetic abundance of the butanoate pathway was observed in children with ERA. Children with ERA also had increased abundance of B. fragilis. Notably, adults with longstanding $\mathrm{SpA}$ had similar trends with respect to $F$. prausnitzii abundance, but opposite trends with respect to Bacteroides, with decreased abundance of Bacteroides genus seen in adult SpA patients compared to adult healthy controls.

This work supports a potential role of $F$. prausnitzii in the pathogenesis of SpA. Although previous studies assessing the microbiota in adults with SpA have not reported alterations in the abundance of this organism [5, 6, 43], this was reported previously in a pediatric study of $\mathrm{SpA}$ patients [8]. This particular species is generally considered to have anti-inflammatory effects through production of SCFAs, such as butyrate [10], or by direct effects on cytokine production [39], and our results demonstrated decreased genetic capacity to synthesize butyrate among ERA patients compared to healthy control pediatric subjects. SCFAs serve as major sources of energy for the intestinal enterocytes and also regulate the differentiation of $\mathrm{T}$ cells, promoting a regulatory phenotype $[46,48]$. A meta-analysis of studies of patients with IBD showed decreased intestinal abundance of $F$. prausnitzii [9]. Our work extends these previous observations, by showing that the specific strain within F. prausnitzii may be of particular relevance. Recent studies indicated that the A2-165 strain, as compared to L2/6 and other strains, may have greater capacity to induce IL-10 production [40] and has increased promoter activity at the butyryl-CoA CoA transferase gene, which codes for the enzyme that catalyzes the final step in the butyrate synthesis pathway [41]. Thus, not only did ERA patients demonstrate less representation of the butanoate pathway, but their promoter activity at a key locus determining butyrate production may also be diminished. Not surprisingly in light of these results, a study of fecal metabolomics demonstrated diminished representation of the butanoate pathway in ERA patients compared to controls [11].

Another important finding in this work pertained to the abundance of Bacteroides. Consistent with previous observations in children with multiple forms of JIA including ERA [7, 8, 13], this organism was relatively overrepresented among pediatric SpA patients. This finding appears to be uniquely associated with pediatric subjects; several studies in adults with both RA [14, 15, 49] and SpA [5, 6] 
have shown decreased abundance of Bacteroides in patients compared to healthy controls. Similar to these other reports, adult SpA patients in this study showed decreased abundance of Bacteroides.

One potential way to reconcile these findings is that $B$. fragilis may contribute to JIA not by directly causing an inflammatory process, but rather through its effects on the ontogeny of the immune system. Vatanen et al. [50] compared the fecal microbiota of children up to age 3 years in areas with high (Estonia and Finland) and low (Russia) incidences of pediatric autoimmunity, finding among the differences decreased fecal abundance of Bacteroides, particularly Bacteroides dorei, in the Russian children. That the differences in the incidence of autoimmunity may have been related to the abundance of Bacteroides was suggested by findings that the lipopolysaccharide (LPS) of $B$. dorei generated a less robust inflammatory reaction than did that of Escherichia coli yet failed to induce tolerance to repeated doses of endotoxin; furthermore, the LPS of $B$. dorei, unlike that of $E$. coli, did not prevent development of type 1 diabetes when injected intraperitoneally into young nonobese diabetic mice. Thus, elevated fecal abundance of Bacteroides may represent an illustration of the hygiene hypothesis, which posits that abnormal early life microbial exposures result in altered immune priming and consequent increased risk of autoimmune diseases [51].

The findings of our study need to be interpreted in view of its limitations. Despite including subjects from around the country, the sample size was small, and we cannot exclude geographic contributions to the findings. HLA-B27 itself may also have contributed to the variance [52], although among the ERA patients there was no evident clustering based upon the presence versus absence of this marker, and there is to our knowledge no published data indicating that the HLA-B27 allele affects the microbiota in humans. In addition, all adult participants with longstanding SpA had been exposed to a variety of immunosuppressive therapies. Furthermore, three of them had been exposed to sulfasalazine, which is in part an antibiotic; however, excluding them did not alter our findings. We also do not have information on subclinical intestinal inflammation, which is frequently present in patients with SpA [53]. Finally, many of the adult and pediatric controls had been referred to evaluate for arthritis; however, these subjects were evaluated by an attending pediatric or adult rheumatologist, who excluded a diagnosis of arthritis on the basis of the historical and physical examination findings. The strengths of the study are that the pediatric arm was limited to treatment-naïve subjects, we used a novel informatics approach to identify strain-level differences between patients and controls, and we included shotgun sequencing data that corroborated the $16 \mathrm{~S}$ findings.

\section{Conclusion}

Our study supports previous work indicating that decreased fecal abundance of a regulatory strain of $F$. prausnitzii may be at least partly responsible for the pathogenesis of SpA, possibly due to decreased production of butyrate, and that efforts to replenish it in patients with SpA may be a potential therapeutic avenue. In contrast, to the extent that increased abundance of Bacteroides or B. fragilis in children reflects altered immunologic development rather than direct pathogenicity or the organism, enthusiasm for microbial-based interventions to address this organism may be tempered. Instead, our findings may underscore the necessity for prevention efforts, such as avoiding unnecessary use of antibiotics in healthy children $[54,55]$.

\section{Additional file}

Additional file 1: Code for processing of 165 sequences with DADA2 and for shotgun sequence analysis with HUMAnN2. (DOCX 12 kb)

\section{Abbreviations}

ASAS: Assessment of Spondyloarthritis International Society; BLAST: Basic Local Alignment Search Tool; CASPAR: Classification Criteria for Psoriatic Arthritis; DADA: Divisive Amplicon Denoising Algorithm; DIAMOND: double index alignment of next-generation sequencing data; ERA: Enthesitis-related arthritis; FDR: False discovery rate; HUMAnN: HMP Unified Metabolic Analysis; IBD: Inflammatory bowel disease; JIA: Juvenile idiopathic arthritis; KEGG: Kyoto Encyclopedia of Genes and Genomes; LPS: Lipopolysaccharide; PCOA: Principal coordinates analysis; PERMANOVA: Permutation multivariate analysis of variance; PSA: Psoriatic arthritis; QIIME: Quantitative Insight Into Microbial Ecology; RA: Rheumatoid arthritis; rDNA: Ribosomal DNA; SCFA: Short chain fatty acids; UAB: University of Alabama at Birmingham

\section{Acknowledgements}

Not applicable.

\section{Funding}

MLS was supported by the Childhood Arthritis and Rheumatology Research Alliance, the American College of Rheumatology, and the NIH-NIAMS P60 AR064172. PAN was supported by the Fundación Bechara. MID was supported by NIH-NIAMS K23 AR062100. In addition, the following are acknowledged for their support of the Microbiome Resource at the University of Alabama at Birmingham: School of Medicine, Comprehensive Cancer Center (P30 CA013148), Center for AIDS Research (5P30AI027767), Center for Clinica Translational Science (UL1TR001417), and Heflin Center for Genomic Sciences.

Availability of data and materials

The fastq files supporting the conclusions of this article will be deposited in the Sequence Read Archive upon publication.

\section{Authors' contributions \\ MLS designed the study, performed bioinformatics and statistical analyses, and drafted the manuscript. PFW, JEW, PAN, BSE, CHS, MGP, KS, and AR were all PIs at their respective institutions and contributed data on one or more subjects reported herein. MID and SLB recruited adult patients at UAB. RQC supervised all aspects of the study and provided substantial input into the initial draft. CDM runs the Microbiome facility at UAB and oversaw the collection and processing of the samples. RK and EJL met with MLS on a regular basis to provide informatics support. All authors read and approved the final manuscript.}

Ethics approval and consent to participate

This study was approved by the Office of the IRB at UAB as well as at the respective study sites: Children's Hospital of Philadelphia, Hackensack University Medical Center, Children's Hospital Boston, Connecticut Children's 
Medical Center, Nationwide Children's Hospital, University of Texas at Southwestern Medical Center (which governs Texas Scottish Rite Hospital), University of Louisville, and Children's Hospital of LA. Informed consent and assent as appropriate were obtained from all study subjects and/or their guardians as per local regulations.

\section{Consent for publication}

Not applicable.

\section{Competing interests}

The authors declare that they have no competing interests.

\section{Publisher's Note}

Springer Nature remains neutral with regard to jurisdictional claims in published maps and institutional affiliations.

\section{Author details}

${ }^{1}$ University of Alabama at Birmingham, Birmingham, AL, USA. ${ }^{2}$ Children's Hospital of Philadelphia, Philadelphia, PA, USA. ${ }^{3}$ Hackensack University Medical Center, Hackensack, NJ, USA. ${ }^{4}$ Boston Children's Hospital and Brigham and Women's Hospital, Boston, MA, USA. ${ }^{5}$ Connecticut Children's Medical Center, Hartford, CT, USA. ${ }^{6}$ Nationwide Children's Hospital, Columbus, OH, USA. ${ }^{7}$ Texas Scottish Rite Hospital, Dallas, TX, USA. ${ }^{8}$ University of Louisville, Louisville, KY, USA. ${ }^{9}$ Children's Hospital of Los Angeles, Los Angeles, CA, USA.

Received: 7 August 2017 Accepted: 2 January 2018

Published online: 30 January 2018

\section{References}

1. Stoll ML. Gut microbes, immunity, and spondyloarthritis. Clin Immunol. 2015;159(2):134-42.

2. Sheehan D, Shanahan F. The gut microbiota in inflammatory bowel disease. Gastroenterol Clin North Am. 2017;46(1):143-54.

3. Hansen JJ, Sartor RB. Therapeutic manipulation of the microbiome in IBD: current results and future approaches. Curr Treat Options Gastroenterol. 2015;13(1):105-20.

4. van Sommeren S, Janse M, Karjalainen J, Fehrmann R, Franke L, Fu J, et al. Extraintestinal manifestations and complications in inflammatory bowel disease: from shared genetics to shared biological pathways. Inflamm Bowel Dis. 2014;20(6):987-94.

5. Stebbings S, Munro K, Simon MA, Tannock G, Highton J, Harmsen H, et al. Comparison of the faecal microflora of patients with ankylosing spondylitis and controls using molecular methods of analysis. Rheumatology (Oxford). 2002;41(12):1395-401.

6. Tito RY, Cypers H, Joossens M, Varkas G, Van Praet L, Glorieus E, et al. Brief Report: Dialister as a microbial marker of disease activity in spondyloarthritis. Arthritis Rheumatol. 2017:69(1):114-21.

7. Aggarwal A, Sarangi AN, Gaur P, Shukla A, Aggarwal R. Gut microbiome in children with enthesitis-related arthritis in a developing country and the effect of probiotic administration. Clin Exp Immunol. 2017;187(3):480-9.

8. Stoll ML, Kumar R, Morrow CD, Lefkowitz EJ, Cui X, Genin A, et al. Altered microbiota associated with abnormal humoral immune responses to commensal organisms in enthesitis-related arthritis. Arthritis Res Ther. 2014;16(6):486.

9. Cao Y, Shen J, Ran ZH. Association between Faecalibacterium prausnitzii reduction and inflammatory bowel disease: a meta-analysis and systematic review of the literature. Gastroenterol Res Pract. 2014;2014:872725.

10. Hold GL, Schwiertz A, Aminov RI, Blaut M, Flint HJ. Oligonucleotide probes that detect quantitatively significant groups of butyrate-producing bacteria in human feces. Appl Environ Microbiol. 2003;69(7):4320-4.

11. Stoll ML, Kumar R, Lefkowitz EJ, Cron RQ, Morrow CD, Barnes S. Fecal metabolomics in pediatric spondyloarthritis implicate decreased metabolic diversity and altered tryptophan metabolism as pathogenic factors. Genes Immun. 2016;17(7):400-5.

12. Muller PH, De Meij TG, Westedt M, De Groot EF, Allaart CF, Brinkman DM, et al. Disturbance of microbial core species in new-onset juvenile idiopathic arthritis. J Pediatr Infect Dis. 2017:12:131-5.

13. Tejesvi MV, Arvonen M, Kangas SM, Keskitalo PL, Pirttila AM, Karttunen TJ, et al. Faecal microbiome in new-onset juvenile idiopathic arthritis. Eur J Clin Microbiol Infect Dis. 2016;35(3):363-70.
14. Scher JU, Sczesnak A, Longman RS, Segata N, Ubeda C, Bielski C, et al. Expansion of intestinal Prevotella copri correlates with enhanced susceptibility to arthritis. Elife. 2013;2:e01202.

15. Maeda Y, Kurakawa T, Umemoto E, Motooka D, Ito Y, Gotoh K, et al. Dysbiosis contributes to arthritis development via activation of autoreactive T cells in the intestine. Arthritis Rheumatol. 2016;68(11):2646-661.

16. Martin R, Miquel S, Benevides L, Bridonneau C, Robert V, Hudault S, et al. Functional characterization of novel Faecalibacterium prausnitzii strains isolated from healthy volunteers: a step forward in the use of F prausnitzii as a next-generation probiotic. Front Microbiol. 2017;8:1226.

17. Ahern PP, Faith JJ, Gordon Jl. Mining the human gut microbiota for effector strains that shape the immune system. Immunity. 2014;40(6):815-23.

18. De Filippo C, Cavalieri D, Di Paola M, Ramazzotti M, Poullet JB, Massart S, et al. Impact of diet in shaping gut microbiota revealed by a comparative study in children from Europe and rural Africa. Proc Natl Acad Sci U S A. 2010;107(33):14691-6.

19. Zhang J, Guo Z, Xue Z, Sun Z, Zhang M, Wang L, et al. A phylo-functional core of gut microbiota in healthy young Chinese cohorts across lifestyles, geography and ethnicities. ISME J. 2015;9(9):1979-90.

20. Petty RE, Southwood TR, Manners P, Baum J, Glass DN, Goldenberg J, et al International League of Associations for Rheumatology classification of juvenile idiopathic arthritis: second revision, Edmonton, 2001. J Rheumatol. 2004;31(2):390-2.

21. Rudwaleit M, van der Heijde D, Landewe R, Listing J, Akkoc N, Brandt J, et al. The development of Assessment of SpondyloArthritis International Society classification criteria for axial spondyloarthritis (part II): validation and final selection. Ann Rheum Dis. 2009;68(6):777-83.

22. Rudwaleit M, van der Heijde D, Landewe R, Akkoc N, Brandt J, Chou CT, et al. The Assessment of SpondyloArthritis International Society classification criteria for peripheral spondyloarthritis and for spondyloarthritis in general. Ann Rheum Dis. 2011;70(1):25-31.

23. Taylor W, Gladman D, Helliwell P, Marchesoni A, Mease P, Mielants H. Classification criteria for psoriatic arthritis: development of new criteria from a large international study. Arthritis Rheum. 2006;54(8):2665-73.

24. Callahan BJ, McMurdie PJ, Rosen MJ, Han AW, Johnson AJ, Holmes SP. DADA2: high-resolution sample inference from Illumina amplicon data. Nat Methods. 2016;13(7):581-3.

25. Rosen MJ, Callahan BJ, Fisher DS, Holmes SP. Denoising PCR-amplified metagenome data. BMC Bioinformatics. 2012;13:283.

26. Caporaso JG, Kuczynski J, Stombaugh J, Bittinger K, Bushman FD, Costello EK, et al. QIIME allows analysis of high-throughput community sequencing data. Nat Methods. 2010;7(5):335-6.

27. Schloss PD, Westcott SL, Ryabin T, Hall JR, Hartmann M, Hollister EB, et al. Introducing mothur: open-source, platform-independent, communitysupported software for describing and comparing microbial communities. Appl Environ Microbiol. 2009;75(23):7537-41.

28. Wang Q, Garrity GM, Tiedje JM, Cole JR. Naive Bayesian classifier for rapid assignment of rRNA sequences into the new bacterial taxonomy. Appl Environ Microbiol. 2007;73(16):5261-7.

29. DeSantis TZ, Hugenholtz P, Larsen N, Rojas M, Brodie EL, Keller K, et al. Greengenes, a chimera-checked 16S rRNA gene database and workbench compatible with ARB. Appl Environ Microbiol. 2006;72(7):5069-72.

30. Schmieder R, Edwards R. Quality control and preprocessing of metagenomic datasets. Bioinformatics. 2011;27(6):863-4.

31. Langmead B, Salzberg SL. Fast gapped-read alignment with Bowtie 2. Nat Methods. 2012;9(4):357-9.

32. Abubucker S, Segata N, Goll J, Schubert AM, Izard J, Cantarel BL, et al. Metabolic reconstruction for metagenomic data and its application to the human microbiome. PLoS Comput Biol. 2012;8(6):e1002358.

33. Pundir S, Martin MJ, O'Donovan C. UniProt Protein Knowledgebase. Methods Mol Biol. 2017;1558:41-55.

34. Buchfink B, Xie C, Huson DH. Fast and sensitive protein alignment using DIAMOND. Nat Methods. 2015;12(1):59-60.

35. Caspi $R$, Altman $T$, Billington $R$, Dreher $K$, Foerster $H$, Fulcher $C A$, et al. The MetaCyc database of metabolic pathways and enzymes and the BioCyc collection of Pathway/Genome Databases. Nucleic Acids Res. 2014; 42(Database issue):D459-71.

36. Kanehisa M, Sato $Y$, Kawashima M, Furumichi M, Tanabe M. KEGG as a reference resource for gene and protein annotation. Nucleic Acids Res. 2016;44(D1):D457-62.

37. Kelly BJ, Gross R, Bittinger K, Sherrill-Mix S, Lewis JD, Collman RG, et al. Power and sample-size estimation for microbiome studies using pairwise distances and PERMANOVA. Bioinformatics. 2015;31(15):2461-8. 
38. Benjamini Y, Hochberg Y. Controlling the false discovery rate: a practical and powerful appraoch to multiple testing. J R Stat Soc B. 1995;57:289-300.

39. Sokol H, Pigneur B, Watterlot L, Lakhdari O, Bermudez-Humaran LG, Gratadoux JJ, et al. Faecalibacterium prausnitzii is an anti-inflammatory commensal bacterium identified by gut microbiota analysis of Crohn disease patients. Proc Natl Acad Sci U S A. 2008;105(43):16731-6.

40. Rossi $\mathrm{O}$, van Berkel LA, Chain F, Tanweer Khan M, Taverne N, Sokol H, et al. Faecalibacterium prausnitzii A2-165 has a high capacity to induce IL-10 in human and murine dendritic cells and modulates T cell responses. Sci Rep. 2016;6:18507.

41. Song H, Yoo Y, Hwang J, Na YC, Kim HS. Faecalibacterium prausnitzii subspecies-level dysbiosis in the human gut microbiome underlying atopic dermatitis. J Allergy Clin Immunol. 2016;137(3):852-60.

42. Round JL, Lee SM, Li J, Tran G, Jabri B, Chatila TA, et al. The Toll-like receptor 2 pathway establishes colonization by a commensal of the human microbiota. Science. 2011;332(6032):974-7.

43. Costello ME, Ciccia F, Willner D, Warrington N, Robinson PC, Gardiner B, et al. Intestinal dysbiosis in ankylosing spondylitis. Arthritis Rheumatol. 2015;67:686-91.

44. Breban M, Tap J, Leboime A, Said-Nahal R, Langella P, Chiocchia G, et al. Faecal microbiota study reveals specific dysbiosis in spondyloarthritis. Ann Rheum Dis. 2017;76(9):1614-622.

45. Turnbaugh PJ, Hamady M, Yatsunenko T, Cantarel BL, Duncan A, Ley RE, et al. A core gut microbiome in obese and lean twins. Nature. 2009;457(7228): 480-4.

46. Hamer HM, Jonkers D, Venema K, Vanhoutvin S, Troost FJ, Brummer RJ. Review article: the role of butyrate on colonic function. Aliment Pharmacol Ther. 2008;27(2):104-19.

47. Taurog JD, Richardson JA, Croft JT, Simmons WA, Zhou M, Fernandez-Sueiro JL, et al. The germfree state prevents development of gut and joint inflammatory disease in HLA-B27 transgenic rats. J Exp Med. 1994;180(6):2359-64.

48. Segain JP, Raingeard De La Bletiere D, Bourreille A, Leray V, Gervois N, Rosales C, et al. Butyrate inhibits inflammatory responses through NFkappaB inhibition: implications for Crohn's disease. Gut. 2000:47(3):397-403.

49. Vaahtovuo J, Munukka E, Korkeamaki M, Luukkainen R, Toivanen P. Fecal microbiota in early rheumatoid arthritis. J Rheumatol. 2008;35(8):1500-5.

50. Vatanen T, Kostic AD, d'Hennezel E, Siljander H, Franzosa EA, Yassour M, et al. Variation in microbiome LPS immunogenicity contributes to autoimmunity in humans. Cell. 2016;165(4):842-53.

51. Rook GA. Hygiene hypothesis and autoimmune diseases. Clin Rev Allergy Immunol. 2012;42(1):5-15.

52. Lin $P$, Bach M, Asquith $M$, Lee AY, Akileswaran L, Stauffer $P$, et al. HLA-B27 and human beta2-microglobulin affect the gut microbiota of transgenic rats. PLoS One. 2014;9(8):e105684.

53. Mielants H, De Keyser F, Baeten D, Van den Bosch F. Gut inflammation in the spondyloarthropathies. Curr Rheumatol Rep. 2005;7(3):188-94.

54. Arvonen M, Virta LJ, Pokka T, Kroger L, Vahasalo P. Repeated exposure to antibiotics in infancy: a predisposing factor for juvenile idiopathic arthritis or a sign of this group's greater susceptibility to infections? J Rheumatol. 2015;42(3):521-6.

55. Horton DB, Scott Fl, Haynes K, Putt ME, Rose CD, Lewis JD, et al. Antibiotic exposure and juvenile idiopathic arthritis: a case-control study. Pediatrics. 2015;136(2):e333-43.

\section{Submit your next manuscript to BioMed Central and we will help you at every step:}

- We accept pre-submission inquiries

- Our selector tool helps you to find the most relevant journal

- We provide round the clock customer support

- Convenient online submission

- Thorough peer review

- Inclusion in PubMed and all major indexing services

- Maximum visibility for your research

Submit your manuscript at www.biomedcentral.com/submit

) Biomed Central 